\title{
Correction: Exploring nutrition knowledge and dietary intake of adults with spinal cord injury in specialist rehabilitation
}

Priya lyer · Eleanor J. Beck · Karen L. Walton

Published online: 21 August 2020

(c) International Spinal Cord Society 2020

Correction to: Spinal Cord 58:930-938

https://doi.org/10.1038/s41393-020-0430-x

published online 11 February 2020

Following publication of this article, the authors noticed some errors in Table 1. The percentages for all the comorbidities, and diets, excluding "full (regular diets with no restriction)", were erroneous. This table has now been

Table 1 General characteristics as $n(\%)$.

\begin{tabular}{llc}
\hline Participant demographics & & $n(\%)$ \\
\hline Gender & Male & $35(70)$ \\
& Female & $15(30)$ \\
Age (years) & $18-50$ years & $25(50)$ \\
& $>50$ years & $25(50)$ \\
Injury level & Paraplegia & $24(48)$ \\
& Tetraplegia & $26(52)$ \\
Injury type & Complete & $13(26)$ \\
& Incomplete & $37(74)$ \\
Time since injury & $1.5-2$ months & $19(38)$ \\
& 3 months & $18(36)$ \\
Education & $4-5$ months & $13(26)$ \\
Family structure & Secondary School/Trade/Technical & $39(78)$ \\
Anthropometry (based on level of injury) & Delow HWR ${ }^{\Delta}$ & $11(22)$ \\
& Within HWR & $21(42)$ \\
& Single, separated/divorced or widowed & $29(58)$ \\
Co-morbidities & Above HWR & $2(4)$ \\
& Type 2 Diabetes Mellitus & $19(38)$ \\
& Hypertension & $29(58)$ \\
& Heart disease & $5(2.5)$ \\
& Hypercholesterolemia & $12(6)$ \\
Diet codes during admission & Depression & $7(3.5)$ \\
& Osteoporosis & $3(1.5)$ \\
& Obstructive Sleep Apnoea & $3(1.5)$ \\
& Chronic Obstructive Pulmonary & $2(1)$ \\
& Disease & $2(1)$ \\
& Full (regular diets with no restriction $)$ & $1(0.5)$ \\
& Diabetes diet, with no set carbohydrate & $42(84)$ \\
& Vegetarian diet with milk and eggs & $2(1)$ \\
& Soft diet & $1(0.5)$ \\
& & \\
& & \\
& &
\end{tabular}

${ }^{\Delta}$ Healthy weight range. replaced with a corrected version. The original, incorrect table is displayed below for reference.

In the results section of the paper, the percentage of participants with hypertension was also incorrectly given as $6 \%$. This has now been corrected to $24 \%$.

This has been corrected in both the PDF and HTML versions of the article. 